\title{
ADAPTAÇÃO ABERTA: AVALIAÇÃO DA SATISFAÇÃO DOS USUÁRIOS DE UM CENTRO DE ALTA COMPLEXIDADE
}

\section{Open fitting: evaluating user satisfaction in a high complexity center}

\author{
Mirela Machado Picolini (1), Wanderléia Quinhoeiro Blasca ${ }^{(2)}$, \\ Karis de Campos ${ }^{(3)}$, Maria Fernanda Capoani Garcia Mondelli (4)
}

\begin{abstract}
RESUMO
Objetivo: avaliar subjetivamente o grau de satisfação dos usuários de AASI open fit (OTE). Método: 11 indivíduos com deficiência auditiva neurossensorial, de grau leve a severo, com configuração descendente, usuários de AASI OTE, foram submetidos ao questionário de auto-avaliação International Outcome Inventory for Hearing Aids (IOI-HA - versão em Português). Resultados: em relação aos sete domínios avaliados, verificou-se que a média referente ao uso foi de 4,45; quanto ao benefício foi de 4,27; em relação à limitação de atividade residual foi de 4,45; quanto à satisfação foi de 4,72; quanto à restrição de participação de atividade residual foi de 4,81; para o domínio impacto nos outros foi de 4,90 e 4,18 para a qualidade de vida. Ressalta-se que a pontuação máxima para cada domínio avaliado era de 5,0 e a mínima 1,0. A média obtida para na pontuação total (soma de todas as questões) foi de 31,81 sendo a máxima 35,0 . Na comparação entre os fatores, houve diferença estatística significante do Fator 2 sobre o Fator 1. Conclusão: com este estudo pode-se atestar o alto grau de satisfação do uso do AASI OTE apresentado pela maioria da amostra coletada, em todos os domínios analisados.
\end{abstract}

DESCRITORES: Auxiliares de Audição; Reabilitação; Qualidade de Vida; Perda Auditiva

\section{INTRODUÇÃO}

O aparelho de amplificação sonora individual (AASI) representa um recurso tecnológico de extrema importância para a reabilitação da deficiência auditiva1.

(1) Fonoaudióloga; Aluna de Pós-Graduação da Faculdade de Odontologia de Bauru da Universidade de São Paulo, FOB/USP, Bauru (SP); Mestranda em Fonoaudiologia na Faculdade de Odontologia de Bauru da Universidade de São Paulo.

(2) Fonoaudióloga; Professora do Departamento de Fonoaudiologia da Faculdade de Odontologia de Bauru da Universidade de São Paulo, FOB/USP, Bauru (SP); Doutora em Distúrbios da Comunicação pelo Hospital de Reabilitação de Anomalias Craniofaciais.

(3) Fonoaudióloga; Mestranda em Fonoaudiologia na Faculdade de Odontologia de Bauru da Universidade de São Paulo.

(4) Fonoaudióloga; Professora do Departamento de Fonoaudiologia da Faculdade de Odontologia de Bauru da Universidade de São Paulo, FOB/USP, Bauru (SP); Doutora em Distúrbios da Comunicação pelo Hospital de Reabilitação de Anomalias Craniofaciais.

Conflito de interesses: inexistente
Mesmo com os avanços tecnológicos, miniaturização dos aparelhos, aperfeiçoamento das características eletroacústicas e melhoria da qualidade sonora, o processo de seleção do AASI deve ser individualizado, levando em consideração entre outros fatores, a configuração audiológica da perda auditiva.

As perdas auditivas podem ocorrer em algumas regiões específicas da cóclea, causada pela ausência ou diminuição das células ciliadas internas ${ }^{2}$, como a perda auditiva em alta freqüência ${ }^{3}$.

Nos últimos anos, a perda auditiva específica em alta freqüência tem aumentado muito em decorrência da exposição ao ruído, presbiacusia, ototoxicidade, ou a combinação desses fatores ${ }^{3}$. Neste caso, os indivíduos apresentam audição normal nas freqüências baixas $(250,500,1000 \mathrm{~Hz})$ e perda auditiva somente nas freqüências altas (3000 $8000 \mathrm{~Hz})$.

A adaptação do AASI em perdas auditivas com esta configuração audiométrica, ou seja, com freqüências baixas preservadas pode ser problemática. Em geral, os indivíduos adaptados com 
AASI retroauricular convencional (BTE) referem dificuldade de inteligibilidade de fala em situações de ruído, autofonia e efeito de oclusão ${ }^{4}$.

Visando atender, minimizar e suprir as necessidades auditivas destes indivíduos, os fabricantes de aparelhos auditivos desenvolveram o AASI denominado "open fit" (OTE). Os AASI OTE ou adaptação aberta são aparelhos auditivos mini-retroauriculares, conectados ao conduto auditivo externo por meio de um tubo e de um domus de silicone ${ }^{5}$. Sua indicação é restrita a indivíduos que necessariamente apresentam audição normal para as baixas freqüências e perda de grau leve a moderado nas freqüências altas, enfatizando a amplificação especificamente essa região de freqüência ${ }^{6}$.

Os usuários de AASI OTE geralmente referem alto nível de benefício e satisfação ${ }^{5,7}$, correlacionado ao fato deste sistema de amplificação apresentar mínimo ou ausente efeito de oclusão, sistemas de cancelamento de feedback acústico e microfones direcionais para melhora da compreensão de fala em situação de ruído 8 .

Apesar dos avanços tecnológicos dos sistemas de amplificação sonora, a satisfação do usuário continua sendo um desafio para os audiologistas e as altas taxas de abandono do uso da prótese auditiva um problema para os serviços de saúde $^{9}$. O bom êxito do processo de adaptação do AASI depende, entre outros fatores, da satisfação do indivíduo com os resultados do uso da sua prótese auditiva ${ }^{10}$. Dessa forma, corresponder às expectativas e necessidades do usuário é o desfecho principal do processo de reabilitação.

O presente estudo teve como objetivo avaliar a satisfação do indivíduo proporcionada pelo uso do AASI OTE, por meio da aplicação de um questionário de auto-avaliação.

\section{MÉTODO}

Este estudo prospectivo foi desenvolvido na Clínica de Fonoaudiologia da Faculdade de Odontologia de Bauru da Universidade de São Paulo (FOB/USP), sendo esta credenciada no Sistema Único de Saúde (SUS) para oferecer serviços de Atenção à Saúde Auditiva em alta complexidade.

Participaram desta pesquisa 11 indivíduos sendo 3 do sexo feminino e 8 do sexo masculino.

Foram considerados como critérios de inclusão:

- indivíduos com diagnóstico audiológico de perda auditiva com configuração audiométrica descendente, tipo neurossensorial de grau entre leve a severo

- usuários de AASI OTE

- indivíduos regularmente matriculados da Clínica de Fonoaudiologia da FOB/USP

Foram excluídos os pacientes com perda auditiva mista, com curvas ascendentes ou planas de qualquer grau.

O grau de perda auditiva foi classificado utilizando os limiares audiométricos das freqüências de $500,1000,2000$ e $4000 \mathrm{~Hz}$, sendo considerada perda auditiva de grau leve (média de 26 a 40 dBNA), moderada (média de 41 a $60 \mathrm{dBNA}$ ), severa (média de 61 a $80 \mathrm{dBNA}$ ) e profunda (média acima de $81 \mathrm{dBNA}$ ), segundo a $\mathrm{WHO}^{11}$

Todos os participantes do estudo foram voluntários, tendo assinado o termo de consentimento livre e esclarecido. Na tabela 1 encontra-se a caracterização dos participantes no que se refere à idade, gênero, classificação socioeconômica, dados da entrevista, adaptação, média das orelhas direita e esquerda e tempo de adaptação no momento em que o estudo foi realizado.

\section{Tabela 1 - Caracterização dos participantes}

\begin{tabular}{|c|c|c|c|c|c|c|c|c|}
\hline & Idade & Gênero & $\begin{array}{c}\text { Classificação } \\
\text { socioeconômica }\end{array}$ & $\begin{array}{c}\text { Dados relevantes } \\
\text { da entrevista }\end{array}$ & Adaptação & $\begin{array}{c}\text { Média } \\
\text { OD }\end{array}$ & $\begin{array}{c}\text { Média } \\
\text { OE }\end{array}$ & $\begin{array}{l}\text { Tempo de } \\
\text { adaptação }\end{array}$ \\
\hline SI & 50 & $\mathrm{M}$ & baixa superior & exposição à ruído & $1^{\mathrm{a}}$ adapt & 25 & 22,5 & 4 meses \\
\hline S2 & 81 & $M$ & média inferior & $\begin{array}{c}\text { medicação } \\
\text { ototóxica }\end{array}$ & $1^{\mathrm{a}}$ adapt & 38,7 & 37,5 & 2 meses \\
\hline S3 & 70 & M & baixa superior & exposição à ruído & $1^{\mathrm{a}}$ adapt & 38,7 & 32,5 & 3 meses \\
\hline S4 & 66 & $\mathrm{~F}$ & baixa superior & - & $1^{\mathrm{a}}$ adapt & 40 & 41,2 & 3 meses \\
\hline S5 & 78 & $M$ & média inferior & - & $1^{\mathrm{a}}$ adapt & 48,7 & 50 & 2 meses \\
\hline S6 & 44 & $\mathrm{~F}$ & média inferior & DA familial & reposição (BTE) & 32,5 & 33,7 & 5 meses \\
\hline S7 & 61 & $M$ & baixa superior & exposição à ruído & $1^{\mathrm{a}}$ adapt & 46,2 & 56,2 & 6 meses \\
\hline S8 & 70 & $\mathrm{~F}$ & baixa superior & - & $1^{\mathrm{a}}$ adapt & 43,7 & 50 & 6 meses \\
\hline s9 & 68 & $M$ & baixa superior & exposição à ruído & $1^{\mathrm{a}}$ adapt & 28,7 & 21,2 & 5 meses \\
\hline S10 & 75 & $M$ & baixa superior & - & reposição (BTE) & 38,7 & 48,7 & 5 meses \\
\hline S11 & 62 & $M$ & média inferior & exposição à ruído & $1^{\mathrm{a}}$ adapt & 40,1 & 51,2 & 5 meses \\
\hline
\end{tabular}

Legenda: OD: orelha direita; OE: orelha esquerda 
A satisfação do usuário foi avaliada de forma subjetiva por meio do questionário de auto-avaliação International Outcome Inventory for Hearing Aids $(\mathrm{IOI}-\mathrm{HA})^{12}$, traduzido para o português ${ }^{13}$. O questionário é composto por oito questões e avalia sete domínios que verificam o resultado da adaptação quanto: uso diário, benefício, limitação de atividades residuais, satisfação, restrição de participação residual, impacto nos outros e qualidade de vida. Portanto, o IOI-HA avalia sob a visão do próprio usuário de AASI sua evolução no uso deste dispositivo nos aspectos mencionados.

Estes domínios foram avaliados na condição com o AASI OTE, após o usuário ter tido uma experiência mínima de dois meses com a amplificação. Cada pergunta tem cinco alternativas que são graduadas do pior desempenho ao melhor desempenho obtido pelo usuário naquele domínio. Se o usuário assinalar o pior desempenho, receberá 1 ponto e se assinalar o melhor desempenho para aquele domínio receberá 5 pontos.

Além da pontuação para cada questão, também foi analisada a pontuação total, que pode ser no máximo 35 e mínima 7 , e a pontuação considerando o Fator 1 (questões 1, 2, 4 e 7) que analisa a interação do indivíduo com seu AASI; e o Fator 2 (questões 3,5 e 6 ) que verifica a interação do indivíduo com as pessoas que estão inseridas em seu ambiente de convívio. O Fator 1 totaliza 4 pontos no mínimo e 20 no máximo e o Fator 2 totaliza 3 pontos no mínimo e 15 no máximo. Dessa forma, quanto maior a pontuação obtida, melhor o desempenho em relação ao AASI.

Este estudo foi submetido e aprovado pelo Comitê de Ética em Pesquisa em Seres Humanos da Faculdade de Odontologia de Bauru sob parecer n¹60/2009.

Para analisar os resultados do questionário IOI-HA foi realizada a análise estatística descritiva da pontuação obtida em cada domínio, da pontuação total e dos Fatores 1 e 2. A comparação da pontuação obtida nos Fatores 1 e 2 foi analisada por meio do Teste T de Student, com nível de significância de $p<0,05$.

\section{RESULTADOS}

$\mathrm{Na}$ primeira questão do questionário IOI-HA, referente ao domínio uso, a figura 1 mostra que $54,5 \%$ da amostra relataram permanecer com o AASI entre 4 e 8 horas por dia, enquanto que $45,4 \%$ permanecem mais que $8 \mathrm{~h}$ por dia. Na tabela 2 pode-se verificar que a pontuação média obtida para este domínio foi de 4,45.

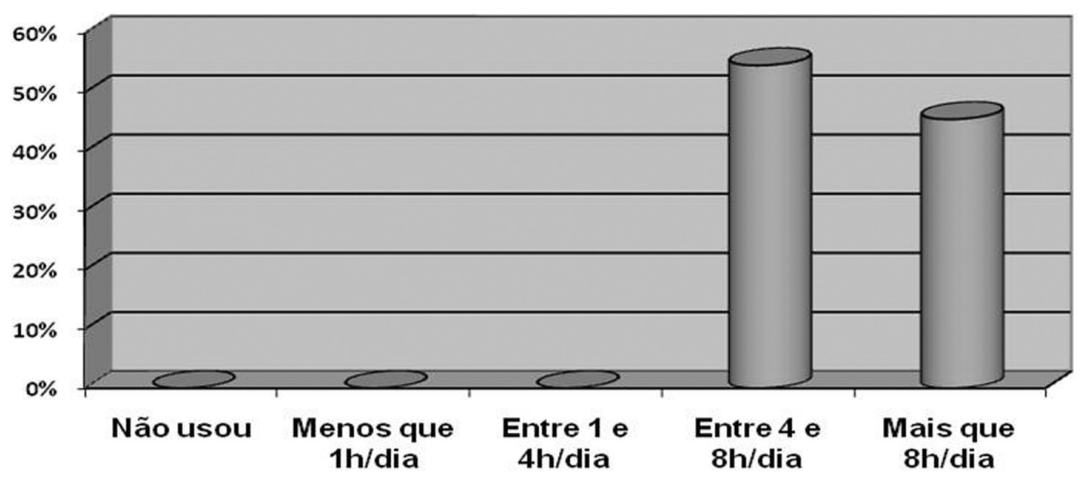

口Questão 1: Pense no tempo em que usou o(s) seu(s) AASI (s) nas últimas duas semanas. Durante quantas horas usou o(s) AASI (s) num dia normal?

Figura 1 - Porcentagem relativa ao domínio uso.

Tabela 2 - Resultados dos sete domínios avaliados do questionário IOI-HA

\begin{tabular}{ccccccccc}
\hline \multicolumn{8}{c}{ Domínios do IOI-HÁ } \\
\hline & Uso & Benefício & LAR & Satisfação & RPR & IO & QV & TOTAL \\
\hline Média & 4,45 & 4,27 & 4,45 & 4,72 & 4,81 & 4,90 & 4,18 & 31,81 \\
Mediana & 4,00 & 4,00 & 5,00 & 5,00 & 5,00 & 5,00 & 5,00 & 32 \\
DP & 0,52 & 0,78 & 0,82 & 0,46 & 0,60 & 0,30 & 1,07 & 3,15 \\
Mínimo & 4,00 & 3,00 & 3,00 & 4,00 & 3,00 & 4,00 & 2,00 & 26 \\
Máximo & 5,00 & 5,00 & 5,00 & 5,00 & 5,00 & 5,00 & 5,00 & 35 \\
\hline
\end{tabular}

Legenda: DP: Desvio padrão; LAR: Limitação de Atividade Residual; RPR: Restrição de Participação Residual; IO: Impacto nos outros e QV: Qualidade de vida 
$\mathrm{Na}$ segunda questão, em relação ao domínio benefício, $45,4 \%$ dos participantes mencionaram que o AASI OTE ajudou muito, $36,3 \%$ que ajudou bastante, $9,1 \%$ que ajudou moderadamente e $9,1 \%$ que ajudou pouco, como demonstrado na figura 2 . A tabela 2 mostra que a pontuação média para este domínio foi de 4,27.

Em relação à terceira questão, relativa ao domínio limitação de atividade residual, $63,6 \%$ da amostra coletada relatou não apresentar dificuldade com o uso do AASI OTE, 18,2\% indicaram que ainda apresentam pouca dificuldade e 18,2\% mencionaram dificuldade moderada. A pontuação média para este domínio foi de 4,45 , como mostra a tabela 2.

$\mathrm{Na}$ figura 4, referente à quarta questão, em relação ao domínio satisfação, pode-se verificar que $72,7 \%$ dos participantes relataram que vale muito a pena utilizar o AASI OTE e $27,2 \%$ disseram valer bastante a pena. A pontuação média referente a este domínio, segundo consta na tabela 2 , foi de 4,72 .

No que diz respeito à quinta questão, demonstrada na figura 5 , abordando o domínio restrição de participação de atividades, 90,9\% dos participantes relataram que os problemas de ouvir não afetaram suas atividades e $9,1 \%$ referiram que tais problemas afetaram moderadamente. Em relação ao domínio a pontuação média obtida foi de 4,81 , como indicado na tabela 2.

$\mathrm{Na}$ figura 6 , correspondente à sexta questão, em relação ao domínio impacto nos outros, 90,9\% da amostra referiu que seus problemas em ouvir não afetaram outras pessoas, enquanto que $9,1 \%$ relataram que esses problemas afetaram pouco os outros. A tabela 2 mostra que para este domínio a pontuação média foi de 4,90.

Na sétima questão, correspondente à figura 7 , abordando o domínio qualidade de vida $54,5 \%$ dos participantes relataram que o AASI OTE aumentou muito mais a alegria de viver, $18,2 \%$ que aumentou bastante a alegria de viver, $18,2 \%$ que aumentou um pouco mais a alegria de viver e 9,1\% mencionaram não ter havido alteração. Para o domínio, nesta questão, a pontuação média obtida foi de 4,18 , como indicado na tabela 2.

$\mathrm{Na}$ tabela 2, segue a pontuação obtida em cada domínio avaliado, com os valores da média, mediana, desvio padrão, pontuação mínima e máxima. Ressalta-se que a média da pontuação total, soma de todos os domínios, verificada na amostra foi de 31,81 o que demonstra um alto nível de satisfação com o AASI OTE, visto que a pontuação total estipulada pelo questionário é de 35 pontos.

Na tabela 3 está apresentada a pontuação dos Fatores 1 e 2, com os valores da média, mediana, desvio padrão, pontuação mínima e máxima. 0 Fator 1 analisa a interação do indivíduo com seu AASI, dado pelas questões 1, 2, 4 e 7, referentes ao uso, benefício, satisfação e qualidade de vida, obtivemos a pontuação média de 17,63. Para o Fator 2, que se atrela à relação do usuário com o ambiente em que ele está inserido, representadas pelas questões 3, 5 e 6 sobre limitação de atividade residual, restrição de participação residual e impacto nos outros, a pontuação média resultante foi de 14,18 .

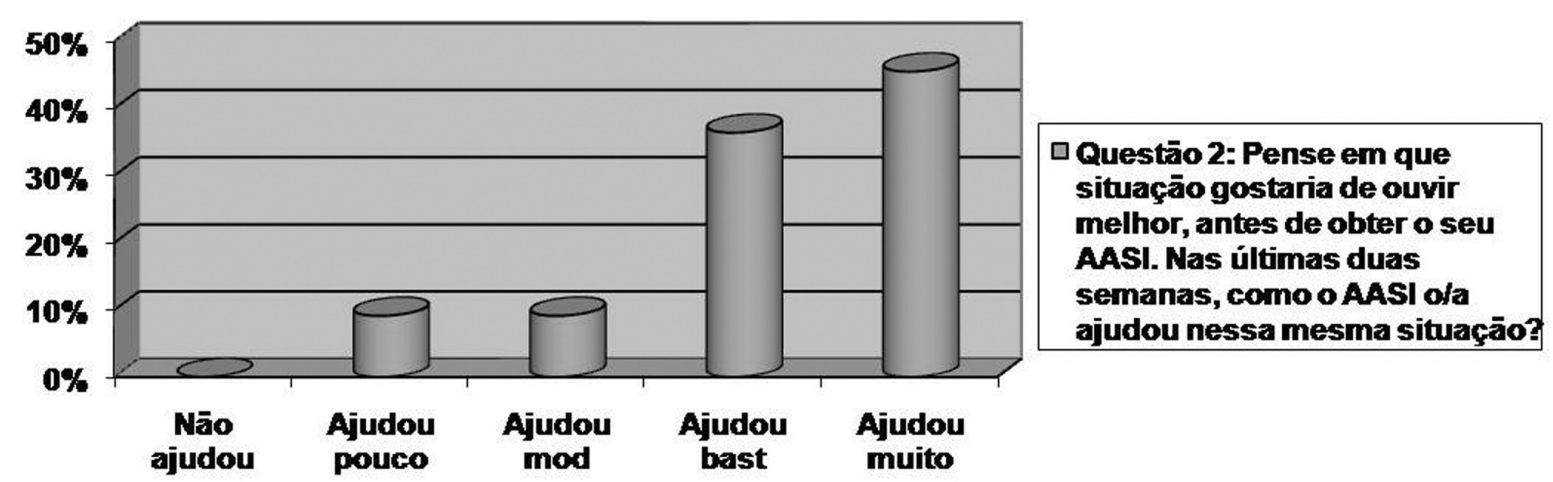

Figura 2 - Porcentagem relativa ao domínio benefício 


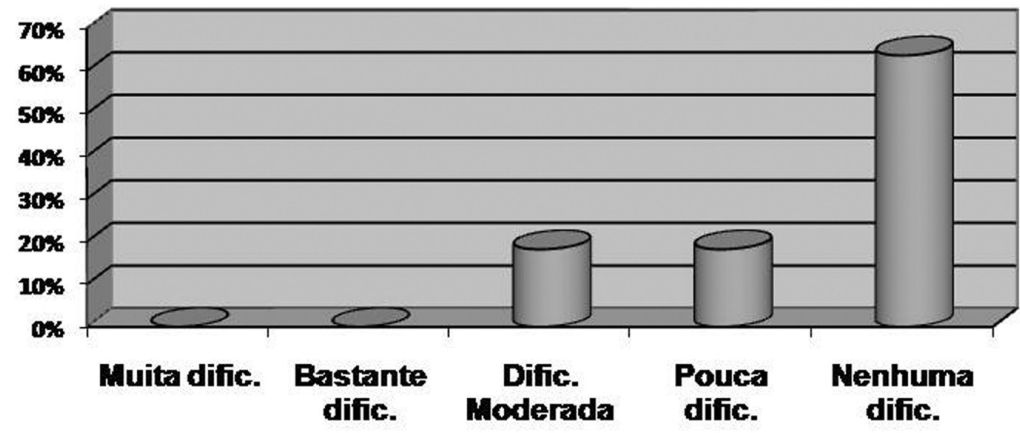

口Questāo 3: Pense novamente na mesma situaçāo em que gostaria de ouvir melhor, antes de obter o(s) seu(s) AASI(s). Que grau de dificuldade AINDA encontra nessa mesma situaçāo usando o(s) AASI (s)?

Figura 3 - Porcentagem relativa ao domínio limitação de atividade residual

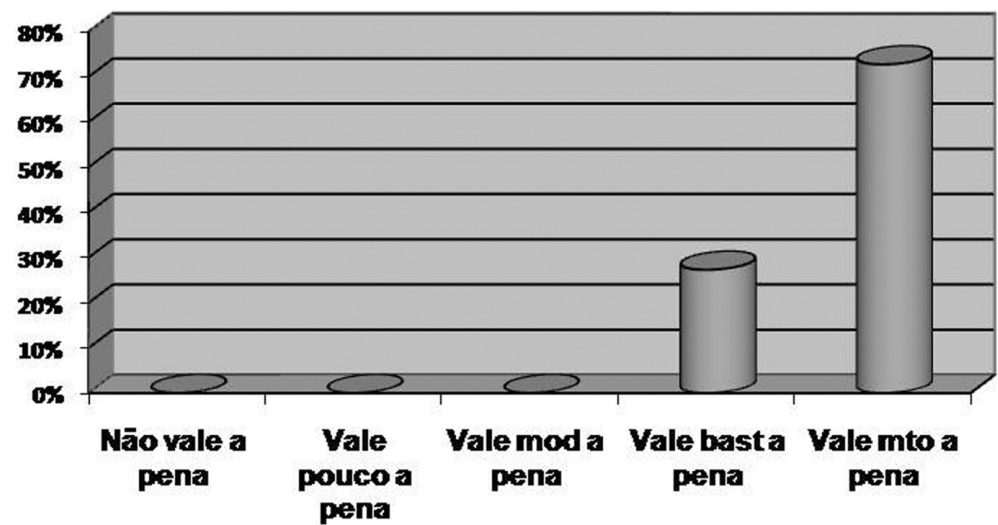

口Questāo 4: Considerando tudo, acha que vale a pena usar o (s) AASI (s)?

Figura 4 - Porcentagem relativa ao domínio satisfação

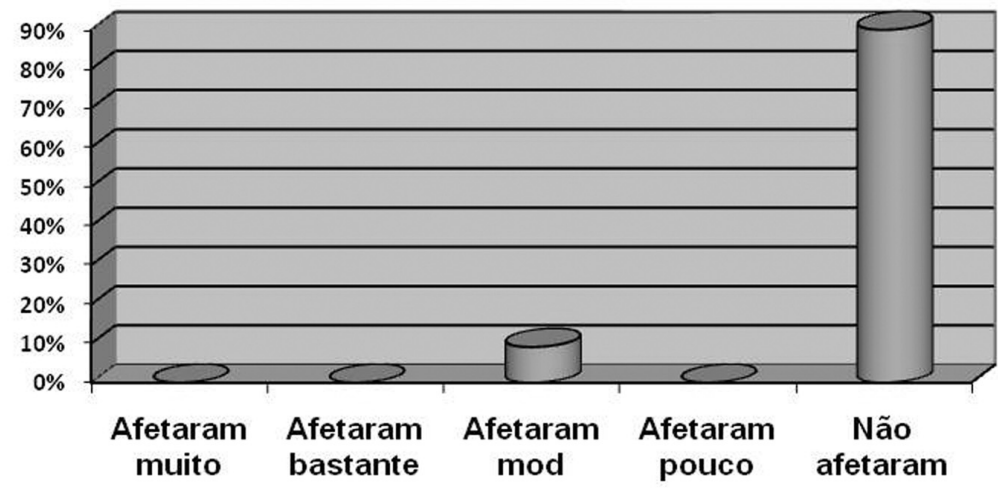

पQuestão 5: Pense nas últimas duas semanas, usando o(s) AASI (s)? Quanto os seus problemas de ouvir o/a afetaram nas suas atividades?

Figura 5 - Porcentagem relativa ao domínio restrição de participação residual

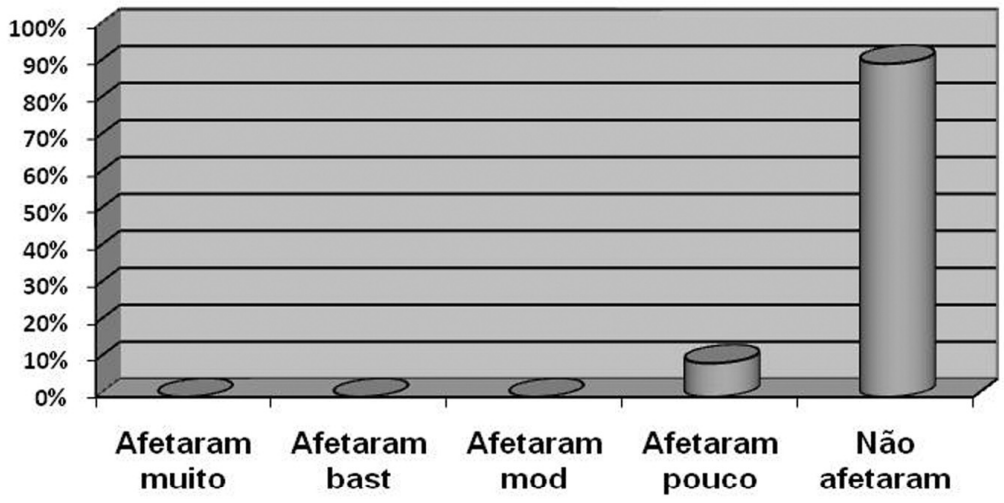

$\square$ Questão 6: Pense nas últimas duas semanas, usando o(s) AASI (s). Quanto os seus problemas de ouvir afetaram ou aborreceram outras pessoas?

Figura 6 - Porcentagem relativa ao domínio impacto nos outros 


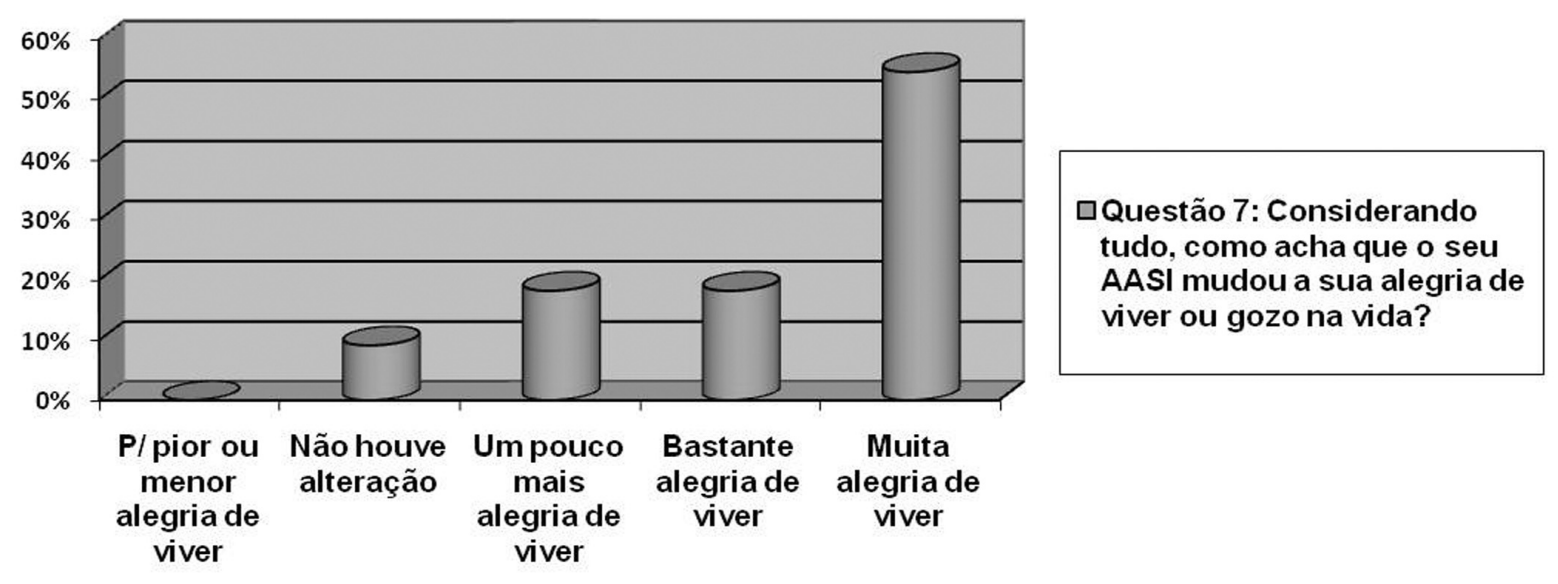

Figura 7 - Porcentagem relativa ao domínio qualidade de vida

Tabela 3 - Resultados dos Fatores 1 e 2 e da pontuação total do questionário IOI-HA

\begin{tabular}{cccc}
\hline & Fator $\mathbf{1}$ & Fator $\mathbf{2}$ & TOTAL \\
\hline Média & 17,63 & 14,18 & 31,81 \\
Mediana & 18 & 15 & 32 \\
DP & 2,24 & 1,07 & 3,15 \\
Mínimo & 14 & 12 & 26 \\
Máximo & 20 & 15 & 35 \\
\hline
\end{tabular}

Legenda: DP: Desvio padrão

\section{DISCUSSÃO}

No início da década de 90, o atendimento ao deficiente auditivo foi incorporado pelo Sistema Único de Saúde (SUS). No ano de 2004, o Ministério da Saúde institui a Política Nacional de Atenção à Saúde Auditiva por meio da portaria GM/MS no 2073/2004 ${ }^{14}$. De acordo com as diretrizes desta nova política, ficou estabelecido pela portaria SAS/ MS no 587/2004 ${ }^{15}$ que os centros de média e alta complexidade devem assegurar um processo de adaptação do AASI que contemple desde a seleção das características físicas e acústicas do molde auricular, como também as características do AASI. Além disso, estabeleceu-se que no processo de validação da adaptação do AASI devem ser aplicados protocolos de percepção de fala e questionários de avaliação do benefício e satisfação do paciente e/ou família ${ }^{16}$.

Dessa forma, a satisfação do usuário, está intimamente relacionada com o sucesso da reabilitação. Outro estudo ${ }^{17}$ também ressalta a importância de verificar a satisfação dos usuários de AASI em Serviços de Atenção à Saúde Auditiva credenciados no SUS.
Analisando os dados da tabela $1,72,7 \%$ dos indivíduos eram do sexo masculino e $27,3 \%$ feminino, com idades variando entre 44 e 81 anos (média de 65,9 anos). De acordo com a OMS, considerando idosas as pessoas acima de 60 anos, este estudo demonstra uma amostra predominantemente de indivíduos idosos $81,9 \%$.

Dentre as conseqüências do processo natural do envelhecimento, podemos citar as alterações auditivas, que ocorrem principalmente em função dos distúrbios somáticos que atingem a orelha interna, degenerando o órgão auditivo ${ }^{18}$. A perda da sensibilidade auditiva no idoso é conhecida como presbiacusia, sendo caracterizada por uma perda auditiva neurossensorial bilateral em alta freqüência ${ }^{19}$.

As informações relatadas na entrevista sobre a história clínica das alterações auditivas e a perda auditiva em alta freqüência predominante na amostra, nos permitem correlacionar à perda auditiva em alta freqüência com a presbiacusia em $36,3 \%$ da amostra, com a exposição a ruído em $45,5 \%$, em relação à ototoxidade em $9 \%$ e a combinação desses fatores em $45,4 \%$. Estes achados corroboram com a literatura consultada 19-22.

Quanto à classificação socioeconômica, 63,3\% da amostra pertence ao estrato social baixo 
superior, reflexo não só de outro estudo ${ }^{23}$ realizado em um centro de alta complexidade da saúde auditiva, como também da realidade brasileira.

Em relação ao tempo de adaptação do AASI OTE, a variabilidade entre os indivíduos estendeuse de 2 meses a 6 meses. $O$ efeito de aclimatização que sucede à adaptação do AASI evidencia uma melhora nas habilidades de reconhecimento de fala e conseqüentemente na comunicação e satisfação do usuário. Alguns autores ${ }^{24}$ não verificaram uma correlação entre o período de aclimatização e a satisfação do usuário de AASI por meio do questionário IOI-HA. Entretanto, verificou-se que não houve insatisfação independente do tempo de adaptação, reflexo da alta pontuação obtida na aplicação deste questionário. Estes achados são concordantes com nosso estudo, uma vez que verificamos uma média obtida de 31,81 para a pontuação total quando a máxima é de 35 pontos.

$\mathrm{Na}$ Tabela 2, em relação à pontuação total obtida pelos usuários de AASI OTE, foi verificada uma média de 31,81 , variando de 26,0 a 35,0 , sendo que a pontuação total máxima é de 35,0 . Em relação aos domínios avaliados pelo questionário, $54,5 \%$ referiram utilizar o AASI entre 4 e 8 horas por dia, $45,4 \%$ relataram que o AASI ajudou muito, $63,6 \%$ referiram não apresentar dificuldade auditiva com a AASI, $72,7 \%$ acham que vale muito a pena utilizar o AASI, 90,9\% relataram que os problemas de ouvir não afetaram suas atividades, 90,9\% referiram que seus problemas em ouvir não afetaram outras pessoas e $54,5 \%$ acham que o AASI aumentou muito mais sua alegria de viver.Diante destes resultados, verifica-se um alto grau de satisfação dos usuários de AASI OTE.

Outras pesquisas também avaliaram o resultado da adaptação por meio do questionário IOI-HA. Não foi encontrada diferença significativa da satisfação do usuário quando comparada com a amplificação monoaural e binaural ${ }^{25}$, tecnologia analógica e digital ${ }^{26}$ e amplificação linear e não-linear ${ }^{27}$. No entanto, usuários de AASI OTE quando comparados a usuários de AASI BTE, apresentam maior benefício e satisfação7.

$\mathrm{Na}$ Tabela 3 foi verificada a relação do indivíduo com seu AASI (Fator 1) e com seu meio ambiente (Fator 2), sendo que a alta pontuação desses fatores, 17,63 (pontuação máxima de 20) e 14,18 (pontuação máxima de 15) respectivamente, refletiu bons resultados da adaptação, assim como em outros estudos ${ }^{27,28}$. Na comparação do Fator 1 e 2 , foi encontrada uma diferença estatisticamente significante $(p=0,016)$, indicando que a amostra demonstrou uma melhor relação do indivíduo com o meio em que ela está inserido. Tal resultado pode indicar que a adaptação do AASI OTE é capaz de reinserir o usuário no convívio com a sociedade, uma vez que, no processo de envelhecimento, a deficiência auditiva, dentre as privações sensoriais, é a que produz maior impacto na comunicação do indivíduo idoso (maior parte da amostra deste estudo), levando-o ao isolamento ${ }^{29}$.

No contexto deste estudo, a aplicação dos questionários de auto-avaliação são procedimentos rápidos, simples e eficientes para avaliar o benefício, satisfação e o sucesso da amplificação. A avaliação da satisfação obtida com o uso do AASI deve ser impreterivelmente subjetiva, pois envolve fatores relacionados à expectativa do usuário, custos monetários e psicossociais, aos problemas encontrados ao longo do processo de reabilitação e as dificuldades de comunicação ainda existentes ${ }^{30}$.

A aplicação de questionários subjetivos faz-se extremamente necessária na etapa de validação, avaliando a influência da amplificação sonora na vida do usuário, no âmbito social, educacional e emocional. Dessa forma, estes instrumentos de avaliação auxiliam no acompanhamento e reabilitação dos usuários de AASI, pois podem identificar as dificuldades em situações de vida diária, proporcionando uma melhoria na qualidade de vida do deficiente auditivo.

\section{CONCLUSÃO}

Com este estudo pode-se atestar o alto grau de satisfação do uso do AASI OTE apresentado pela maioria da amostra coletada, em todos os domínios analisados (uso diário, benefício, limitação de atividades residuais, satisfação, restrição de participação residual, impacto nos outros e qualidade de vida). No que diz respeito à inter-relação do usuário com o seu AASI OTE (Fator 1) e à interação do indivíduo ao meio em que ele está inserido (Fator 2) houve uma alta pontuação, indicativa de grande satisfação com o uso deste dispositivo

Os avanços tecnológicos, como a adaptação aberta, é uma alternativa viável para indivíduos com perda auditiva em alta freqüência e a escassez de estudos nesta temática evidencia a necessidade de outras pesquisas que abordem detalhadamente o beneficio e a satisfação do usuário de AASI open fit. 


\section{ABSTRACT}

Purpose: to subjectively evaluate the satisfaction degree of users as for the open fit hearing aids (OTE). Method: 11 subjects with neurossensorial hearing impairment, with mild to severe degree, with downward sloping, users of OTE, was applied to the self-assessment International Outcome Inventory for Hearing Aids - (IOI-HA - Portuguese version). Results: in the seven assessed domains, it was found that the average for the daily use was 4.45 , and the benefit was 4.27 , And when compared to residual activity limitation it was 4.45 , the satisfaction level 4.72 , the residual participation restriction was 4.81 , for the impact on others 4.90 and 4.18 for quality of life. It is noteworthy to stress out that the maximum score for each evaluated area was 5.0 and 1.0 as minimum. The average factor for the total score (sum of all questions) was 31.81 and the maximum 35.0. Comparing the factors, we found a statistical significance in Factor 2 over Factor 1. Conclusion: with this study we can demonstrate the high satisfaction degree in using HA OTE submitted by the majority of the sample collected in all analyzed domains.

KEYWORDS: Hearing Aids; Rehabilitation; Quality of Life; Hearing Loss

\section{REFERÊNCIAS BIBLIOGRÁFICAS}

1. Boscolo CC, Costa MPR, Domingos CMP, Perez FC. Avaliação dos benefícios proporcionados pelo AASI em crianças e jovens da faixa etária de 7 a 14 anos. Rev Bras Ed Esp. 2006;12(2):255-68.

2. Moore BC. Dead regions in the cochlea: Conceptual foundations, diagnosis, and clinical applications. Ear Hear. 2004;25(2):98-116.

3. Fabry DA, Launer S, Derleth P. Hearing Aid Technology vs. Steeply Sloping Sensorineural Hearing Loss. Hearing Review [periódico na internet]. Jan 2007 [acesso em 2 de outubro de 2009]. Disponível em: http://www.hearingreview. com/issues/articles/2007-01_04.asp

4. Gnewikow D, Moss M. Hearing aid outcomes with open- and closed-canal fittings. The Hearing Journal. 2006;59(11):66-72.

5. Jespersen CT, Groth J. Can an In-the-Ear Device be Truly Open? Yes!. Hearing Review [periódico na internet]. Aug 2008 [acesso em 2 de outubro de 2009]. Disponível em: http://www.hearingreview. com/issues/articles/2008-08_03.asp

6. Martin RL. Rules for successful open fittings. The Hearing Journal. 2006;59(9):54-9.

7. Taylor B. Real-world satisfaction and benefit with open-canal fittings. The Hearing Journal. 2006;59(11):74-82.

8. Valente M, Mispagel KM. Unaided and aided performance with a directional open fit hearing aid. Int J Audiol. 2008;47(6):329-36.

9. Veiga LR, Merlo ARC, Mengue SS. Satisfação com a prótese auditiva na vida diária em usuários do Sistema de Saúde do Exército. Rev Bras Otorrinolaringol. 2005;71(1):67-73.
10. Teixeira AR, Almeida LG, Jotz GP, Barba MC. Qualidade de vida de adultos e idosos pós adaptação de próteses auditivas. Rev Soc Bras Fonoaudiol. 2008;13(4):357-61.

11. WHO - World Health Organization. Grades of Hearing impairment, 2007 [acesso em 26 de fevereiro de 2009]. Disponível em : http://www.who. int/pbd/desfness/hearing_impairment_grades/en/ index.html

12. Cox RM, Alexander GC. The international outcome inventory for hearing aids (IOI-HA): psychometric properties of the english version. Int $\mathrm{J}$ Audiol. 2002; 41(1):30-5.

13. Cox RM, Stephens D, Kramer, SE. Translation of the international outcome inventory for hearing aids (IOI-HA). Int J Audiol. 2002;41(1):3-26.

14. Ministério da Saúde (a). Portaria GM/MS no. 2.073, de 28 de setembro de 2004. Versa sobre a Política Nacional de Atenção à Saúde Auditiva a ser implantada a todas as unidades federais, respeitadas as competências das três esferas de gestão e de outras providencias. Diário Oficial da União. 29 set 2004; Seção 1:34.

15. Ministério da Saúde (b). Portaria GM/MS no. 587, de 7 de outubro de 2004. Determina que as Secretárias de Estado da Saúde dos Estados adotem as providências necessárias à organização e implantação das redes estaduais de atenção à saúde auditiva. Diário Oficial da União. 11 out 2004; Seção 1:105.

16. Bevilacqua MC, Melo TM, Moretin M, Lopes AC. A avaliação de serviços em audiologia: concepções e perspectivas. Rev Soc Bras Fonoaudiol. 2009;14(3):421-6.

17. Fialho IM, Bortoli D, Mendonça GG, Pagnosim DF, Scholze AS. Percepção de idosos sobre o uso 
de AASI concedido pelo sistema único de saúde. Rev CEFAC. 2009;11(2):338-44.

18. Almeida MR, Guarinello AC. Reabilitação audiológica em pacientes idosos. Rev Soc Bras Fonoaudiol. 2009;14(2):247-55.

19. Silva BSR, Sousa GB, Russo ICP, Silva JAPR. Caracterização das queixas, tipo de perda auditiva e tratamento de indivíduos idosos. Arq Int Otorrino.2007;11(4):387-95.

20. Silva IMC, Feitosa MAG. Audiometria de alta freqüência em adultos jovens e mais velhos quando a audiometria convencional é normal. Rev Bras Otorrinolaringol. 2006;72(5):665-72.

21. Fabry DA. Facts vs Myths: The "Skinny" on Open-Fit Hearing Aids. Hearing Review: International Edition 2007 [periódico na internet]. 2007 [acesso em 2 de outubro de 2009]. Disponível em: http://www.hearingreview.com/issues/articles/ HPR_2007-IN_03.asp

22. Jacob LCB, Aguiar FP, Tomiasi AA, Tschoeke SN, Bitencourt RF. Monitoramento auditivo na ototoxidade. Rev Bras Otorrinolaringol. 2006;72(6):836-44.

23. Morettin M. Avaliação dos benefícios e satisfação dos usuários de Aparelho de Amplificação Sonora Individual nos serviços de audiologia do SUS. [Dissertação], Faculdade de Saúde Publica da Universidade de São Paulo, 2008.
24. Prates LPCS, lório MCM. Aclimatização: estudo do reconhecimento de fala em usuários de próteses auditivas. Pro Fono. 2006;18(3):259-66.

25. Magni C, Armentano JN, Moreira OS, Winter EW. Investigação do grau de satisfação entre usuários de amplificação monoaural e binaural. Distúrbios da Comunicação. 2005;17(3):323-32.

26. Magni C, Freiberger F, Tonn K. Avaliação do grau de satisfação entre os usuários de amplificação de tecnologia analógica e digital. Rev Bras Otorrinolaringol. 2005;71(5):650-7.

27. Costa LP, lório MCM. Próteses auditivas: avaliações objetivas e subjetivas em usuários de amplificação linear e não-linear. Pro Fono. 2006;18(1):21-30.

28. Mello JM, Machado PF, Quintino VV. Questionário IOI-HÁ na versão em português (QI-AASI). Fono Atual. 2005;34(8):65-73.

29. Ruschel CV, Carvalho CR, Guarinello AC. A eficiência de um programa de reabilitação audiológica em idosos com presbiacusia e seus familiares. 2007;12(2):95-8.

30. Almeida K. Avaliação dos Resultados de Intervenção. In: Almeida K, lório MCM. Próteses Auditivas: fundamentos teóricos e aplicações clínicas. 2ed. São Paulo: Lovise; 2003. p. 335-55.

http://dx.doi.org/10.1590/S1516-18462010005000122

RECEBIDO EM: 28/04/2010

ACEITO EM: 26/06/2010

Endereço para correspondência:

Maria Fernanda Capoani Garcia Mondelli

Departamento de Fonoaudiologia da Faculdade

de Odontologia de Bauru da Universidade de São

Paulo (FOB/USP)

Alameda Octávio Pinheiro Brisolla 9-75

Bauru - São Paulo

CEP: 17012-901

E-mail: mfernandamondelli@ hotmail.com 